\title{
COMPOUND MATRIX METHOD FOR EIGENVALUE PROBLEMS IN MULTIPLE CONNECTED DOMAINS
}

\author{
N. R. ANTURKAR, T. C. PAPANASTASIOU AND J. O. WILKES \\ Department of Chemical Engineering, The University of Michigan, Ann Arbor, Michigan 48109, U.S.A.
}

\begin{abstract}
SUMMARY
An algorithm based on a compound matrix method is presented for solving difficult eigenvalue problems of $n$ equation sets in connected domains that are coupled through $(n-1)$ sets of interfacial boundary conditions, when $n$ is an arbitrary number. As an example, a linear stability problem of $n$-layer plane Poiseuille flow is formulated. The resulting Orr-Sommerfeld equations form a set of stiff differential equations at high wavenumbers, which are solved accurately for various combinations of parameters.
\end{abstract}

\section{INTRODUCTION}

Standard shooting techniques for finding eigenvalues of a set of differential equations tend to be inaccurate for stiff systems. The accuracy can be improved dramatically by using a corresponding differential system of a compound matrix, the elements of which are the minors of the solution matrix. The resulting compound matrix method was used by Gilbert and Backus $^{1}$ for elastic wave problems, and by $\mathrm{Ng}$ and Reid ${ }^{2-4}$ and Davey ${ }^{5}$ for two-point boundary value problems and eigenvalue problems of the Orr-Sommerfeld equation. These investigations computed eigenvalues and eigenfunctions with marginal errors where standard shooting methods failed. Yiantsios and Higgins ${ }^{6}$ extended the method to equation sets valid over two connected domains that are coupled through interfacial conditions; in particular, they solved the Orr-Sommerfeld equations for two superposed fluids in plane Poiseuille flow. However, since the eigenvalues are obtained by matching the interfacial boundary conditions, the method is unsuitable for computations in more than two domains connected by interfacial boundary conditions.

Here, the compound matrix method is implemented for $n$ equation sets that are valid over connected domains through $(n-1)$ sets of interfacial boundary conditions, where $n$ is arbitrary. Instead of matching boundary conditions at a particular interface for finding eigenvalues, the integration of the compound differential system proceeds with new initial conditions at an interface for the compound differential system of the next domain. Subsequently, the eigenvalues are computed by matching the boundary conditions at the end of the last domain. This general algorithm for finding eigenpairs is used in the linear stability analysis of $n$-layer, Newtonian, plane Poiseuille flow, for which resulting differential systems are known to be stiff at large wavenumbers.

\footnotetext{
* Present address: Polymer Science Department, Scientific Research Lab, Ford Motor Company, Mail Drop 3198 , Dearborn, MI 48121-2053, U.S.A.
} 


\section{ALGORITHM}

We consider the following $n$ linear homogeneous systems of a differential eigenvalue problem:

$$
\boldsymbol{\phi}_{j}^{\prime}=\mathbf{W}_{j}\left(z_{j}\right) \boldsymbol{\phi}_{j}, \quad 0 \leqslant z_{j} \leqslant h_{j}, \quad j=1,2, \ldots, n
$$

where $\mathbf{W}_{j}\left(z_{j}\right)$ is a $4 \times 4$ matrix, the solution $\phi_{j}$ is a $4 \times 1$ vector, and the prime denotes a derivative with respect to $z_{j}$. The boundary conditions at $z_{1}=0$ and at $z_{n}=h_{n}$ are separated and expressed as

$$
\begin{gathered}
\mathbf{D} \phi_{1}(0)=0 \\
\mathbf{E} \phi_{n}\left(h_{n}\right)=0
\end{gathered}
$$

where $\mathbf{D}$ and $\mathbf{E}$ are $2 \times 4$ matrices. At $(n-1)$ interfaces located at $\left(z_{j}=h_{j}, z_{j+1}=0\right)$, the interfacial boundary conditions are

$$
\mathbf{F}_{j} \boldsymbol{\phi}_{j}\left(h_{j}\right)+\mathbf{G}_{j} \boldsymbol{\phi}_{j+1}(0)=0, \quad j=1,2, \ldots, n-1
$$

where $\mathbf{F}_{j}$ and $\mathbf{G}_{j}$ are $4 \times 4$ matrices. Note that the origin for the $j$ th system is shifted for convenience to the $(j-1)$ th interface.

Let $\phi_{j, 1}$ and $\phi_{j, 2}$ be any two linearly independent solutions, which satisfy the boundary conditions (2) for $j=1$ or the interfacial boundary conditions (4) for $j \neq 1$. The minors of the solution matrix $\left[\phi_{j, 1} \phi_{j, 2}\right]$ are given by

$$
y_{j}(k, l)=\phi_{j, 1 k} \phi_{j, 2 l}-\phi_{j, 1 l} \sigma_{j, 2 k}, \quad k=1,2, \ldots, 3, \quad l=k+1, k+2, \ldots, 4
$$

where $\phi_{j, m k}$ is the $k$ th element of solution vector $\phi_{j, m}$. When $y_{j}(k, l)$ are arranged in the lexicographic order of their indices, e.g. $y_{j}(1,2)=y_{j, 1}, y_{j}(1,3)=y_{j, 2}$ and so on, they form the elements of the second compound matrix $\mathbf{y}_{j}$. These elements satisfy the quadratic identity ${ }^{4}$

$$
y_{j, 1} y_{j, 6}-y_{j, 2} y_{j, 5}+y_{j, 3} y_{j, 4}=0
$$

Schwart $z^{7}$ has shown that the differential compound system for $\mathbf{y}_{j}$ is given by

$$
\mathbf{y}_{j}^{\prime}=\mathbf{H}_{j}\left(z_{j}\right) \mathbf{y}_{j}, \quad j=1,2, \ldots, n
$$

where $\mathbf{H}_{j}\left(z_{j}\right)$ is known in terms of $\mathbf{W}_{j}\left(z_{j}\right)$ such that

$$
\mathbf{H}_{j}\left(z_{j}\right)=\left[\begin{array}{cccccc}
w_{j, 11}+w_{j, 22} & w_{j, 23} & w_{j, 24} & -w_{j, 13} & -w_{j, 14} & 0 \\
w_{j, 32} & w_{j, 11}+w_{j, 33} & w_{j, 34} & -w_{j, 12} & 0 & -w_{j, 14} \\
w_{j, 42} & w_{j, 43} & w_{j, 11}+w_{j, 44} & 0 & w_{j, 12} & w_{j, 13} \\
-w_{j, 31} & w_{j, 21} & 0 & w_{j, 22}+w_{j, 33} & w_{j, 34} & -w_{j, 24} \\
-w_{j, 41} & 0 & w_{j, 21} & w_{j, 43} & w_{j, 22}+w_{j, 44} & w_{j, 23} \\
0 & -w_{j, 41} & w_{j, 31} & -w_{j, 42} & w_{j, 23} & w_{j, 33}+w_{j, 44}
\end{array}\right],
$$

where $w_{j, k l}$ is the $(k, l)$ th element of $\mathbf{W}_{j}$.

From the matrix $\left[\begin{array}{ll}\phi_{1,1} & \phi_{1,2}\end{array}\right]$ at $z_{1}=0$, the initial condition $\mathbf{y}_{1}(0)$ is obtained by using (5). Suppose that $\mathbf{y}_{1}$ is computed by integrating (7) for $j=1$ from $z_{1}=0$ to $h_{1}$ for a given initial estimate of an eigenvalue $c$. The next step in the algorithm is to find $\mathbf{y}_{2}(0)$ by satisfying the interfacial conditions (4) for $j=1$, and then to compute $y_{2}$ by integrating (7) for $j=2$ from $z_{2}=0$ to $h_{2}$. The process continues until every $\mathbf{y}_{j}$ is computed for $j=1,2, \ldots, n$ for a given initial estimate of $c$. The matrix $\mathbf{M}\left(=\mathbf{E}\left[\phi_{n, 1}\left(h_{n}\right) \phi_{n, 2}\left(h_{n}\right)\right]\right)$ is singular for non-trivial $\phi_{j}$, and 
for linearly independent $\phi_{n, 1}\left(h_{n}\right)$ and $\phi_{n, 2}\left(h_{n}\right)$. Therefore, by using (5),

$$
\operatorname{det} \mathbf{M}=\sum_{k=1}^{3} \sum_{l=k+1}^{4}\left(E_{1 k} E_{2 l}-E_{1 l} E_{2 k}\right) \mathbf{y}_{n}(k, l)\left(h_{n}\right)
$$

where $\mathbf{y}_{j}(k, l)$ are defined in (5). Once $\mathbf{y}_{n}$ is calculated for a given estimate of $c$, the eigenvalue relationship (9) is satisfied by varying the eigenvalue $c$ using a suitable iterative procedure.

The crucial step in the above procedure is to find $\mathbf{y}_{j+1}(0)$ by satisfying the interfacial conditions (4) for a known $\mathbf{y}_{j}\left(h_{j}\right)$. This procedure is as explained below. Suppose that $\mathbf{y}_{j}$ is computed by integrating (7) from $z_{j}=0$ to $h_{j}$. Since the solution $\phi_{j}$ of (1) can be expressed as a linear combination of $\phi_{j, 1}$ and $\phi_{j, 2}$, the relationship between $\mathbf{y}_{j}$ and $\phi_{j}$ is derived using (5) as

$$
\mathbf{Q}_{j}\left(\mathbf{y}_{j}\right) \boldsymbol{\phi}_{j}=0
$$

where

$$
\mathbf{Q}_{j}=\left[\begin{array}{cccc}
y_{j, 4} & -y_{j, 2} & y_{j, 1} & 0 \\
y_{j, 5} & -y_{j, 3} & 0 & y_{j, 1} \\
y_{j, 6} & 0 & -y_{j, 3} & y_{j, 2} \\
0 & y_{j, 6} & -y_{j, 5} & y_{j, 4}
\end{array}\right]
$$

However, by using (6) and row reduction on $\mathbf{Q}_{j}$, it can be shown that $\mathbf{Q}_{j}$ has rank 2 . Therefore, two linearly independent solutions $\phi_{j}\left(h_{j}\right)$ are obtained using two equations from $(10)$. The interfacial boundary conditions (4) provide two additional linearly independent solutions of $\phi_{j+1}(0)$. These four equations are used to find $\mathbf{y}_{j+1}(0)$. For non-singular $\mathbf{G}_{j}$, the solutions computed by the above procedure are linearly independent.

After determining $c$, the eigenfunctions are computed. In several engineering applications, however, the eigenfunctions are not needed. In those cases, the subsequent steps can be avoided. For fixed $(k, l)$ with $k \neq l$, two auxiliary equations are obtained from (1) and (5). They are

$$
\begin{aligned}
y_{j}(k, l) \phi_{j, k}^{\prime} & =\sum_{m=1}^{4} w_{j, k m}\left[y_{j}(m, l) \phi_{j, k}-y_{j}(m, k) \phi_{j, l}\right] \\
y_{j}(k, l) \phi_{j, l}= & \sum_{m=1}^{4} w_{j, l m}\left[y_{j}(m, l) \phi_{j, k}-y_{j}(m, k) \phi_{j, l}\right]
\end{aligned}
$$

Once $\mathbf{y}_{j}$ is calculated by using the previous algorithm, two equations taken from $(10)$ and two equations taken from (11) provide the required number of equations for determining $\boldsymbol{\phi}_{j}$, assuming that $y_{j}(k, l)$ does not vanish in the interval $0<z_{j} \leqslant h_{j}$.

The boundary condition $\phi_{n}\left(h_{n}\right)$, required for integrating (11) for $j=n$ from $z_{n}=h_{n}$ to 0 , is provided by simultaneously solving (3) and two linearly independent equations taken from (10). The unique solution is guaranteed for non-singular $\mathbf{E}$. $^{2}$ Subsequently, to integrate (11) for any $j \neq 1$, the interfacial conditions (4) provide the necessary initial conditions $\phi_{j-1}\left(h_{j-1}\right)$, which depend on $\phi_{j}(0)$.

However, a caution is warranted in computing eigenfunctions using (11). In many cases, $z_{j}=0$ is a regular singular point of (11), even in a single domain. ${ }^{2}$ In those cases, the above procedure of backward integration will fail to yield the final values at $z_{j}=0$. However, the algorithm requires $\phi_{j}$ at $z_{j}=0$, because $\phi_{j-1}\left(h_{j-1}\right)$ depends on $\phi_{j}(0)$. If $z_{j}=0$ is a singular point, then $\phi_{j}(0)$ is computed by extrapolating the values of eigenfunctions near $z_{j}=0$ using any standard formula, rather than by solving (11). Besides, with a proper choice of the auxiliary equations, the exponents can be made positive at a singular point, and then the simple 
matching problem from $z_{j}=h_{j}$ to 0 is not expected to yield any numerical difficulties, even near $z_{j}=0$. This important aspect of the algorithm was discussed by $\mathrm{Ng}$ and $\mathrm{Reid}^{2}$ for their calculations on a single domain.

\section{EXAMPLE: LINEAR STABILITY ANALYSIS OF MULTILAYER PLANE POISEUILLE FLOW}

As a test problem, the compound matrix method is implemented in the linear stability analysis of $n$-layer, Newtonian, plane Poiseuille flow. With a convenient co-ordinate transformation shown in Figure 1, the dimensionless velocity profile for the primary flow is ${ }^{8}$

$$
v_{x j}=A_{j}+B_{j} z_{j}+C_{j} z_{j}^{2}, \quad 0 \leqslant z_{j} \leqslant h_{j}, \quad j=1,2, \ldots, n
$$

where $A_{j}, B_{j}$ and $C_{j}$ are the constants, and $h_{j}$ the thickness, which is made dimensionless with respect to the total thickness of the channel. The constants and the thicknesses of all the layers are computed for a given set of flow rates $q_{j}, j=1,2, \ldots, n$, by means of (12). The boundary conditions are no-slip at the walls, and continuity of velocities and stresses at the interfaces.

The linear stability analysis of the primary flow in (12) gives the well-known Orr-Sommerfeld equation for each layer in terms of streamfunctions $\phi_{j}$ of the disturbed velocities. ${ }^{8,9}$ When the Orr-Sommerfeld equations are written in the form of (1), the non-zero elements of $\mathbf{w}_{j}$ are:

$$
\begin{aligned}
& w_{j, 12}=w_{j, 23}=w_{j, 34}=1 \\
& w_{j, 41}=-i \alpha \frac{\operatorname{Re}_{j}}{m_{j}}\left[\alpha^{2}\left(v_{x j}-c\right)+v_{x j}^{\prime \prime}\right]-\alpha^{4} \\
& w_{j, 43}=2 \alpha^{2}+i \alpha \frac{\operatorname{Re} e_{j}}{m_{j}}\left(v_{x j}-c\right)
\end{aligned}
$$

where $\alpha$ is the real wavenumber of the disturbance, $c$ the complex wavespeed, $i$ the unit imaginary number, and $m_{j}$ the viscosity ratio of the $j$ th layer to the first layer. The Reynolds number $R e_{j}$ is defined as $\rho_{j} v H / \mu_{1}$, where $\rho_{j}$ is the density of the $j$ th layer, $\mu_{1}$ the viscosity of the first layer, $V$ the average total velocity at the inlet of the channel, and $H$ is the thickness of the channel.

The compound differential system of (1) is then derived from (7) and (8):

$$
\begin{gathered}
y_{j, 1}^{\prime}=y_{j, 2}, \quad y_{j, 2}^{\prime}=y_{j, 3}+y_{j, 4} \\
y_{j, 3}^{\prime}=w_{j, 43} y_{j, 2}+y_{j, 5}, \quad y_{j, 4}^{\prime}=y_{j, 5} \\
y_{j, 5}^{\prime}=-w_{j, 41} y_{j, 1}+w_{j, 43} y_{j, 4}+y_{j, 6}, \quad y_{j, 6}^{\prime}=-w_{j, 41} y_{j, 2}
\end{gathered}
$$

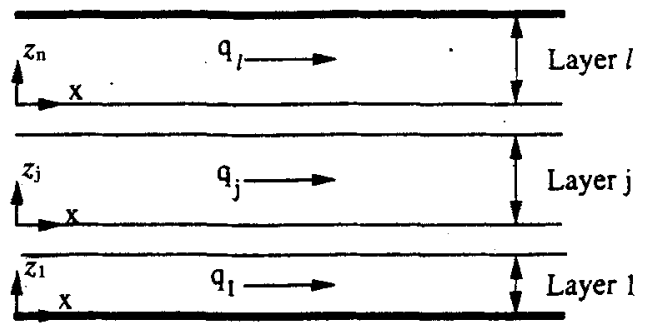

Figure 1. Geometry of multilayer, plane Poiseuille flow and transformation of the co-ordinates 
The no-slip boundary conditions at the walls define the matrices $\mathbf{D}$ and $\mathbf{E}$ to be the unit matrices. The continuity of velocities in the $x$ - and $z$-directions, and the continuity of shear and normal stresses at the interfaces provide the non-zero elements of $\mathbf{F}_{j}$ and $\mathbf{G}_{j}$. They are:

$$
\begin{gathered}
f_{j, 11}=1, \quad f_{j, 21}=\frac{v_{x j}^{\prime}}{c-v_{x j}}, \quad f_{j, 22}=1 \\
f_{j, 31}=m_{j} \alpha^{2}, \quad f_{j, 33}=m_{j} \\
f_{j, 41}=i \alpha R e_{j} v_{x j}^{\prime}+\frac{i \alpha}{c-v_{x j}}\left[\frac{1}{C a_{j}} \alpha^{2}+S t\left(d_{j+1}-d_{j}\right)\right] \\
f_{j, 42}=i \alpha R e_{j}\left(c-v_{x j}\right)-3 \alpha^{2} m_{j}, \quad f_{j, 44}=m_{j}
\end{gathered}
$$

and

$$
\begin{gathered}
g_{j, 11}=-1, \quad g_{j, 21}=-\frac{v_{x(j+1)}^{\prime}}{c-v_{x(j+1)}}, \quad g_{j, 22}=-1 \\
g_{j, 31}=-m_{j+1} \alpha^{2}, \quad g_{j, 33}=-m_{j+1}, \quad g_{j, 41}=-i \alpha R e_{j+1} v_{x(j+1)}^{\prime} \\
g_{j, 42}=-i \alpha R e_{j+1}\left(c-v_{x(j+1)}\right)-3 \alpha^{2} m_{j+1} \\
g_{j, 44}=-m_{j+1}
\end{gathered}
$$

Here, $d_{j}=\rho_{j} / \rho_{1}$ is the density ratio, and (15) and (16) are evaluated at $j=1,2, \ldots,(n-1)$. The steady-state velocity $v_{x j}$ and its derivatives with respect to $z_{j}$ are evaluated at $z_{j}=h_{j}$, whereas the steady-state velocity $v_{x(j+1)}$ and its derivatives with respect to $z_{j+1}$ are evaluated at $z_{j+1}=0$. These derivatives are denoted by primes. The dimensionless groups are the capillary number $C a_{j}=V \mu_{1} / \sigma_{j}$ (inversely proportional to the interfacial tension $\sigma_{j}$ between the $j$ th and $(j+1)$ th layers), and the Stokes number $S t=g h^{2} / v \rho_{1} \mu_{1}$ (proportional to gravity $g$ ).

Let $\phi_{1,1}$ and $\phi_{1,2}$ be two linearly independent solutions of (1) for $j=1$ that satisfy the boundary conditions (2), such that

$$
\phi_{1,1}=[0,0,1,0]^{\mathrm{T}} \quad \text { and } \quad \phi_{1,2}=[0,0,0,1]^{\mathrm{T}}
$$

From (5) and (17),

$$
\mathbf{y}_{1}(0)=[0,0,0,0,0,1]^{\mathrm{T}}
$$

Suppose that $\mathbf{y}_{j}$ is computed by integrating (14) from $z_{j}=0$ to $h_{j}$ following the steps described in Section 2 for an initial estimate of the eigenvalue $c$. The following two equations are then used to find $\mathbf{y}_{j+1}(0)$ from (11):

$$
\begin{aligned}
& y_{j, 6} \phi_{j, 1}-y_{j, 3} \phi_{j, 3}+y_{j, 2} \phi_{j, 4}=0 \\
& y_{j, 6} \phi_{j, 2}-y_{j, 5} \phi_{j, 3}+y_{j, 4} \phi_{j, 4}=0
\end{aligned}
$$

By assuming that $y_{j, 6}$ does not vanish at the boundaries, two linearly independent solutions obtained from (19) at $z_{j}=h_{j}$ are:

$$
\begin{aligned}
\boldsymbol{\phi}_{j, 1} & =\left[\frac{y_{j, 3}}{y_{j, 6}}, \frac{y_{j, 5}}{y_{j, 6}}, 1,0\right] \\
\boldsymbol{\phi}_{j, 2} & =\left[-\frac{y_{j, 4}}{y_{j, 6}},-\frac{y_{j, 2}}{y_{j, 6}}, 0,1\right], \quad j=1,2, \ldots, n-1
\end{aligned}
$$


where $y_{j}$ is evaluated at $z_{j}=h_{j}$. Then $\phi_{j+1}(0)$ is determined using (4), and subsequently, $y_{j+1}(0)$ is evaluated by means of (5). Since $\mathbf{E}$ is the unit matrix,

$$
\operatorname{det} \mathbf{M}=y_{n, 1}=0
$$

The eigenvalue $c$ is computed from (21) by Newton iteration.

When the eigenvalue and $\mathbf{y}_{j}$ over all the connected domains are determined, the eigenfunctions are evaluated from (11). The auxiliary equations are:

$$
\begin{array}{r}
y_{j, 1} \phi_{j}^{\prime \prime}-y_{j, 2} \phi_{j}^{\prime}+y_{j, 4} \phi_{j}=0 \\
y_{j, 1} \phi_{j}^{\prime \prime \prime}-y_{j, 3} \phi_{j}^{\prime}+y_{j, 5} \phi_{j}=0 \\
y_{j, 2} \phi_{j}^{\prime \prime \prime}-y_{j, 3} \phi_{j}^{\prime \prime}+y_{j, 6} \phi_{j}=0 \\
y_{j, 4} \phi_{j}^{\prime \prime \prime}-y_{j, 5} \phi_{j}^{\prime \prime}+y_{j, 6} \phi_{j}^{\prime}=0
\end{array}
$$

Since $y_{j, 1}=0$ at $z_{n}=h_{n},(22 \mathrm{c})$ or (22d) is chosen for backward integration from $z_{n}=h_{n}$ to 0 . The conditions at $z_{n}=h_{n}$ are provided by solving (3) and (19). Note that if $\mathbf{y}_{j}(0)$ does not contain any zero elements (which is the most likely situation), then the limiting behaviour of (22) near $z_{j}=0$ indicates positive exponents, except for $j=1$. The vector $\mathbf{y}_{1}(0)$ does not contain zero elements, and only (22a) has a positive exponent for $j=1$. Therefore, it is necessary to integrate (22a) for the first layer. In this algorithm, $(22 \mathrm{c})$ is integrated in the $n$th layer, and (22a) is integrated in all other layers to obtain the eigenfunctions. The initial conditions for the integration are obtained using the interfacial boundary conditions (4). As mentioned earlier, $z_{1}=0$ is a regular singular point for (22). Therefore, the Newton-Cotes fourth-order extrapolation formula is used near $z_{1}=0$ to get $\phi_{j}(0)$. Since the integration is carried out using the fourth-order Runge-Kutta method, the error estimates of both the integration and extrapolation are of the same order of magnitude.

The calculations were performed on an IBM3090/600E mainframe computer with doubleprecision complex arithmetic. A constant step-size, fourth-order Runge-Kutta method is used for the integration. The Jacobian $\partial y_{n, 1} / \partial c$ in Newton iteration is evaluated numerically. If a suitable initial estimate of $c$ is available, (21) is found to be satisfied in four to five iterations with quadratic convergence within a tolerance limit of $y_{n, 1} \leqslant 10^{-8}$. The drawback of the algorithm is that it can only compute one eigenvalue for a given set of parameters, and depending on the initial estimate the solution may not converge to a leading eigenvalue. However, accurate initial estimates of the leading eigenvalues at small wavenumbers can be obtained from asymptotic solutions, in which case the method converges to the leading mode. The initial estimates in the complete domain of the parameter space are then obtained by the first-order continuation.

The compound matrix method is very efficient at large wavenumbers, where standard shooting techniques fail or do not provide accurate results. Since it is essential to study linear stability over a wide range of wavenumbers for ascertaining the stability of the flow at all wavenumbers, the algorithm is well-suited for linear stability analysis. The asymptotic analysis reveals that multilayer flow can be unstable to interfacial disturbances, even at small Reynolds numbers. We have investigated this interfacial instability, and have carried out all calculations at $R e_{j}<10$, which is typical in many industrial processes of multilayer flows, such as in coating and coextrusion. Owing to the large number of parameters involved in the analysis, the results will be presented here only for two- and three-layer flows. However, the algorithm can be implemented for a flow of any numbers of layers. 


\section{SAMPLE CALCULATION}

The algorithm is validated by comparing numerical results using this algorithm with the asymptotic solutions for $\alpha \rightarrow 0$ for three-layer flow (refer to Table I). The parameters in this calculation are $R e_{j}=5, \quad d_{j}=1 \cdot 0, S t=0, C a_{j}=\infty, m_{2}=20, m_{3}=1$ and $q_{3} / q_{1}=0 \cdot 3$. The wave speeds calculated by the two methods agree well for $\alpha=0.001$ at various values of $q_{2} / q_{1}$, and deviate marginally as $\alpha$ increases from $0 \cdot 001$ to $0 \cdot 1$. Similar calculations are also performed for two-layer flow. The neutral stability curves for two-layer flow also compare well with neutral stability curves at identical conditions in previous studies. ${ }^{8,10}$

An additional set of neutral stability diagrams is presented to emphasize the importance of the algorithm in predicting the interfacial instability at large wavenumbers for a flow with more than two layers. The first set, shown in Figure 2, indicates that the stability of the flow

Table I. A comparison between the asymptotic solutions for $\alpha \rightarrow 0$ and numerical results at small $\alpha$ obtained by the compound matrix method for various $q_{2} / q_{1}$ in three-layer flow at $\operatorname{Re}_{j}=5, C a_{j}=\infty$, $q_{3} / q_{1}=0 \cdot 3, m_{2}=20, m_{3}=1, d_{j}=1$ and $S t=0$

\begin{tabular}{lcc}
\hline$q_{2} / q_{1}$ & 0.5 & $1 \cdot 5$ \\
\hline Asymptotic solution & $1.48455+0.50239 \times 10^{-2} \alpha i$ & $1 \cdot 29088-0 \cdot 11079 \times 10^{-3} \alpha i$ \\
\hline$\alpha=0.001$ & $1.48445+0.50248 \times 10^{-5} i$ & $1 \cdot 29073-0 \cdot 11090 \times 10^{-6} i$ \\
$\alpha=0.01$ & $1.48330+0.50180 \times 10^{-4} i$ & $1 \cdot 29026-0.11162 \times 10^{-5} i$ \\
$\alpha=0.1$ & $1.48106+0.49724 \times 10^{-3} i$ & $1 \cdot 28025-0.12381 \times 10^{-4} i$ \\
\hline$q_{2} / q_{1}$ & \multicolumn{1}{c}{4.0} & \\
\hline Asymptotic solution & $0.99093-0.20649 \times 10^{-2} \alpha i$ & \\
\hline$\alpha=0 \cdot 001$ & $0.99094-0.20630 \times 10^{-5} i$ & \\
$\alpha=0.01$ & $0.99081-0.20589 \times 10^{-4} i$ & \\
$\alpha=0.1$ & $0.98327-0.20346 \times 10^{-3} i$ & \\
\hline
\end{tabular}

(a)

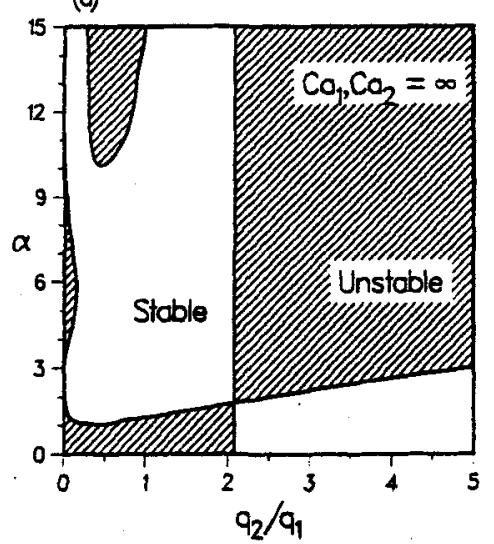

(b)

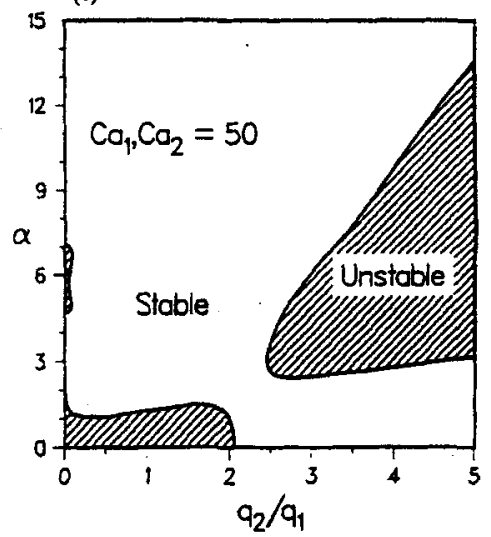

Figure 2. Stabilizing effect of interfacial tensions on the neutral stability curves in three-layer flow in the $\alpha-q_{2} / q_{1}$ plane. The material properties are the same for layers 1 and 3 . The parameters are $m_{2}=20, m_{3}=1, q_{3} \mid q_{1}=0 \cdot 2, d_{j}=1$, $R e_{1}=5$ and $S t=0$. The hatched regions are unstable and the unhatched regions are stable 
improves with decreasing capillary number. In planar flow, disturbances increase the interfacial area. Therefore, interfacial tensions always stabilize the flow by minimizing the interfacial energy through minimization of interfacial area. Therefore, the stabilizing effect, which can be captured efficiently using the compound matrix method, is dramatic at large wavenumbers compared to small wavenumbers. From Figure 2, the critical flow rate ratios within which the flow is stable or unstable at all $\alpha$, can only be estimated when the stability analysis is extended to large wavenumbers. Additional results are presented in Anturkar et al. ${ }^{8}$

\section{SUMMARY}

The advantages of using the compound matrix method for stiff systems (at large wavenumbers and/or at large Reynolds numbers) have already been demonstrated by earlier studies, ${ }^{5,2,4}$ including the study of two-layer flows. ${ }^{6}$ We have shown in this paper that the method can also be implemented for several sets of equations connected through interfacial boundary conditions. The applicability of the algorithm is discussed for cases of hydrodynamic stability analysis of plane Poiseuille flow of more than two layers. Since an iterative technique is used to calculate eigenvalues, the converged numerical solution strongly depends on an initial estimate. Therefore, a major disadvantage of the algorithm is the necessity of providing an appropriate initial estimate to get the most dominant eigenvalue. This problem can be alleviated by complementing the compound matrix method with an approximate asymptotic analysis, or by calculating all the eigenvalues of a discretized problem using standard algorithms for selected parameters.

\section{ACKNOWLEDGEMENT}

This research was funded by a grant from the Dow Chemical Company, Midland, Michigan, whose support is gratefully acknowledged.

\section{REFERENCES}

1. F. Gilbert and G. E. Backus, 'Propagator matrices in elastic wave and vibration problems,' Geophysics, 31, 326-332 (1966).

2. B. S. $\mathrm{Ng}$ and $\mathrm{W}$. H. Reid, 'An initial value method for eigenvalue problems using compound matrices,' J. Comput. Phys., 30, 125-136 (1979).

3. B. S. Ng and W. H. Reid, 'A numerical method for linear two-point boundary-value problems using compound matrices', J. Comput. Phys., 33, 70-85 (1979).

4. B. S. Ng and W. H. Reid, 'The compound matrix method for ordinary differential systems', $J$. Comput. Phys., 58, 209-228 (1985).

5. A. Davey, 'On the numerical solution of difficult boundary-value problems', J. Comput. Phys., 35, $36-47$ (1980).

6. S. G. Yiantsios and B. G. Higgins, 'Numerical solution of eigenvalue problems using the compound matrix method', J. Comput. Phys., 74, 25-40 (1988).

7. B. Schwartz, 'Totally positive differential systems', Pac. J. Math., 32, 203-229 (1970).

8. N. R. Anturkar, T. C. Papanastasiou and J. O. Wilkes, 'Linear stability analysis of multilayer plane Poiseuille flow', Phys. Fluids A, 2, 530 (1990).

9. C. S. Yih, 'Instability due to viscosity stratification', J. Fluid Mech., 27, 337-352 (1967).

10. S. G. Yiantsios and B. G. Higgins, 'Linear stability of plane Poiseuille flow of two superposed fluids: shear and interfacial modes', Phys. Fluids, 31, 3225-3238 (1988). 